\title{
Caenorhabditis elegans rab-3 Mutant Synapses Exhibit Impaired Function and Are Partially Depleted of Vesicles
}

\author{
Michael L. Nonet, ${ }^{1,2}$ Jane E. Staunton, ${ }^{2}$ Michael P. Kilgard, ${ }^{1}$ Tim Fergestad, ${ }^{4}$ Erika Hartwieg, ${ }^{3}$ \\ H. Robert Horvitz, ${ }^{3}$ Erik M. Jorgensen, ${ }^{4}$ and Barbara J. Meyer ${ }^{1}$ \\ ${ }^{1}$ Department of Molecular and Cell Biology, University of California, Berkeley, California 94720, 2Department of Anatomy \\ and Neurobiology, Washington University School of Medicine, St. Louis, Missouri 63110, ${ }^{3}$ Department of Biology and \\ Howard Hughes Medical Institute, Massachusetts Institute of Technology, Cambridge, Massachusetts 02139, and \\ ${ }^{4}$ Department of Biology, University of Utah, Salt Lake City, Utah 84112
}

Rab molecules regulate vesicular trafficking in many different exocytic and endocytic transport pathways in eukaryotic cells. In neurons, rab3 has been proposed to play a crucial role in regulating synaptic vesicle release. To elucidate the role of rab3 in synaptic transmission, we isolated and characterized Caenorhabditis elegans rab-3 mutants. Similar to the mouse rab3A mutants, these mutants survived and exhibited only mild behavioral abnormalities. In contrast to the mouse mutants, synaptic transmission was perturbed in these animals. Extracellular electrophysiological recordings revealed that synaptic transmission in the pharyngeal nervous system was impaired. Furthermore, rab-3 animals were resistant to the acetylcholinesterase inhibitor aldicarb, suggesting that cholinergic transmission was generally depressed. Last, synaptic vesicle populations were redistributed in rab-3 mutants. In motor neurons, vesicle populations at synapses were depleted to $40 \%$ of normal levels, whereas in intersynaptic regions of the axon, vesicle populations were elevated. On the basis of the morphological defects at neuromuscular junctions, we postulate that $\mathrm{RAB}-3$ may regulate recruitment of vesicles to the active zone or sequestration of vesicles near release sites.

Key words: small GTP-binding proteins; exocytosis; rab3; synaptic vesicle proteins; Caenorhabditis elegans; mutants
Calcium-regulated neurotransmitter release at synapses is a specialized form of exocytosis that shares many similarities with other vesicle-mediated secretory processes (Bennett and Scheller, 1994; Sudhof, 1995). One class of molecules that regulates secretory processes is the rab family of small GTP-binding proteins (Simons and Zerial, 1993; Nuoffer and Blach, 1994). Distinct members of this large family of proteins associate with different subcellular compartments of secretory pathways. In particular, members of the rab3 family are associated with synaptic vesicles in neurons and secretory vesicles in neuroendocrine cells (Fischer von Mollard et al., 1994b).

Experiments in Saccharomyces cerevisiae and mouse suggest very different roles for rab molecules in regulating various secretory steps. Genetic analysis of the $S$. cerevisiae rab mutants ypt 1 and sec 4 demonstrates that certain rab molecules are essential for specific vesicular transport steps (for review, see Ferro-Novick and Novick, 1993). Furthermore, biochemical analysis with ypt1 mutant extracts suggests that the formation of biochemical com-

\footnotetext{
Received March 12, 1997; revised Aug. 7, 1997; accepted Aug. 11, 1997.

This research was supported by Grants to B.J.M. from the Muscular Dystrophy Association and to M.L.N. (NS33535) and H.R.H. (GM24663) from the United States Public Health Service (USPHS). M.L.N. was supported during portions of this work by a public service award from the USPHS. H.R.H. is an Investigator for the Howard Hughes Medical Institute. We thank Wayne Davis and Leon Avery for suggestions in setting up the EPG system; Doju Yoshikami and Larry Okun for providing the recording equipment; Jim Thomas for providing programs to aid in assaying defecation; Lisa Chen, Andy Fire, and Jim Kramer for providing strains; and Allison Potter, Liping Wei, and Felisha Starkey for technical assistance. Several C. elegans strains were obtained from the Caenorhabditis Genetics Center (St. Paul, MN).

J.E.S. and M.P.K. contributed equally to this work.

Correspondence should be addressed to Dr. Michael L. Nonet, Department of Anatomy and Neurobiology, Campus Box 8108, Washington University School of Medicine, 660 South Euclid Avenue, St. Louis, MO 63110.

Copyright (C) 1997 Society for Neuroscience $0270-6474 / 97 / 178061-13 \$ 05.00 / 0$
}

plexes between vesicle proteins and proteins on the acceptor membranes requires rab proteins (Lian et al., 1994; Sogaard et al., 1994). These complexes are postulated to be postdocking intermediates in the vesicle fusion process (Sollner and Rothman, 1994). Thus, analyses of yeast rab molecules indicates that rab molecules are essential for either vesicle docking or a step before docking.

Experiments perturbing rab3 function in excitatory cells suggest that this rab plays a more subtle role in regulating release than rab molecules in yeast. Specifically, the mild nature of the secretion defects in rab3A knock-out mice is inconsistent with an essential role for rab3 in regulating transmitter release (Geppert et al., 1994). In these mouse mutants, evoked release is depressed only slightly on repetitive stimulation of hippocampal neuron slices. Although the presence of rab3C on vesicles isolated from brain complicates the interpretation of the rab3A mutant phenotype (Fischer von Mollard et al., 1994a), the mouse studies suggest a regulatory role for rab3 in secretion.

A variety of experiments perturbing rab3 function have proposed both a stimulatory and inhibitory role for rab3 in regulating release. Peptides corresponding to the effector domain of rab3 incubated with permeabilized cells stimulate release in a variety of cells (Oberhause et al., 1992; Padfield et al., 1992; Piiper et al., 1994), suggesting that rab3 inhibits release in the absence of a stimulus. However, the reduction of rab3B levels using antisense oligonucleotides similarly inhibits evoked secretion from pituitary cells (Lledo et al., 1993). Conversely, rab3A antisense oligonucleotides increase evoked release on repetitive stimulation from adrenal chromaffin cells (Holz et al., 1994; Johannes et al., 1994). Finally, recent physiological experiments with cultured mouse hippocampal cells from rab3A mutants suggest that $r a b-3$ negatively regulates a late step in release (Geppert et al., 1997). Thus, 
although perturbation of rab3 activity modulates secretion, its role in regulating the release process remains unclear.

To examine further the role of rab3 in regulating synaptic transmission, we isolated and characterized $r a b-3$ mutants of Caenorhabditis elegans, an organism that expresses only a single rab3 gene. C. elegans mutants deficient in RAB-3 function were slightly deficient in synaptic function because they exhibited mild behavioral defects, physiological abnormalities, and resistance to drugs that potentiate the action of the neurotransmitter acetylcholine. Ultrastructural analysis of synaptic terminals of $r a b-3$ mutant animals revealed that they were depleted of synaptic vesicles by a factor of two- to threefold. The diffuse organization of the remaining vesicles suggested that the primary defects resulted from a reduced ability to retain vesicles near the release site, consistent with either an inability to retain vesicles at synapses or a defect in docking. Although our analysis demonstrated that synaptic function was compromised in $r a b-3$ mutants, RAB-3 clearly was not essential for the release of neurotransmitter.

\section{MATERIALS AND METHODS}

Growth and culture of C. elegans. C. elegans was grown at $20^{\circ} \mathrm{C}$ on solid medium as described by Sulston and Hodgkin (1988). All mapping, complementation, and deficiency testing were performed by standard genetic methods (Herman and Horvitz, 1980). Aldicarb, 2-methyl-2[methylthio]proprionaldehyde $O$-[methylcarbamoyl]oxime, was obtained from Chem Services (West Chester, PA) and was prepared as a $100 \mathrm{~mm}$ stock solution in $70 \%$ ethanol. Aldicarb was added to the agar growth medium after autoclaving or added directly to plates.

DNA and RNA manipulations. C. elegans genomic DNA was isolated as described by Sulston and Hodgkin (1988). cDNA was made by reversetranscribing RNA, using random hexanucleotide primers as described by Sambrook et al. (1989). Poly $\left(\mathrm{A}^{+}\right)$-selected RNA was isolated from a mixed-stage culture of the wild-type strain N2 as previously described (Nonet and Meyer, 1991). Manipulations of DNA and RNA, including electrophoresis, blotting, and probing of blots, were performed by standard procedures, except where noted (Sambrook et al., 1989).

Cloning of $\mathrm{C}$. elegans rab genes. Degenerate oligonucleotides corresponding to regions conserved between the rat and Drosophila rab3 proteins were used to amplify the $C$. elegans gene, using 35 amplification cycles of $45 \mathrm{sec}$ at $94^{\circ} \mathrm{C}, 1 \mathrm{~min}$ at $45^{\circ} \mathrm{C}$, and $2 \mathrm{~min}$ at $72^{\circ} \mathrm{C}$. PCR reactions were performed as described by Innis et al. (1990). oRB-2 (5' GGNGCNATGGGNTTYAT $3^{\prime}$ )/oRB-4 (5'-TCCATRTCRCAYTTRTTNCC$3^{\prime}$ ) products were gel-purified and cloned into pBluescript $\mathrm{KS}\left({ }^{-}\right)$, yielding the plasmid pRB101. pRB101 insert DNA was sequenced and used as a probe to screen four independent pools of an embryonic cDNA library (Miller, 1991). Two alternative transcripts were made from the $r a b-3$ locus. The transcripts differed in whether they included a 63 base $5^{\prime}$ coding exon. Of the cDNAs characterized, 2 of 16 ( $\lambda$ MN14 and $\lambda$ MN15) contained at least a portion of the $5^{\prime}$ coding exon. The cDNA insert of $\lambda \mathrm{MN} 9$ was cloned into pBluescript $\left.\mathrm{KS}^{-}\right)$to create pRB102. cDNA clones of the $5^{\prime}$ end of the message were isolated by a modification of a RACE-PCR method (Innis et al., 1990). Oligonucleotides corresponding to the C. elegans SL1 and SL2 trans-spliced leaders found at the $5^{\prime}$ end of many C. elegans messages (Blumenthal, 1995) and an oligonucleotide complementary to the 5' end of our cDNA (oRB-5; GAATCATCACAGTAACGG) were used to amplify $5^{\prime}$ end cDNA fragments. Two distinct SL1/oRB5-derived PCR products were cloned into pBluescript $\mathrm{KS}\left({ }^{-}\right)$, yielding the plasmids pRB104 and pRB106. The first coding exon was absent from pRB104 and present in pRB106. The pRB102, pRB104, and pRB106 inserts were sequenced. Analysis of the DNA sequence and the deduced amino acid coding sequence of the gene were performed on a SPARC station (Sun Microsystems) with the Genetics Computer Group (GCG) program package (Devereux et al., 1984). The rab-3 cDNA sequences have been deposited into GenBank (accession numbers U68265 and U68266).

Additional $r a b$ genes were isolated from $C$. elegans in three experiments with PCR. Oligonucleotides corresponding to a sequence found in all $r a b$ family members (oRB-30; 5'-GCGGATCCTGGGAYACNGCNGGNCARGA-3') and a sequence found specifically in rab3 (oRB-31; 5'-TCYTGNCCNGCNGTRTCCCANATYTG-3') were used to amplify products from C. elegans cDNA. Products were cloned into pBlue- script $\mathrm{KS}\left({ }^{-}\right)$and sequenced. Ninety-eight $r a b$-3 clones and eight clones encoding a rab8 homolog were isolated by using these oligonucleotides. A second amplification was performed with oRB-31 and an oligonucleotide (oRB-4) corresponding to a sequence found in multiple $r a b$ family members, including rab3. From this PCR reaction we isolated C. elegans clones encoding proteins with high similarity to the following proteins (number of clones isolated is in parentheses): rab1A (10), rab1B (2), rab3 (20), rab8 (40), rab10 (13), rab11(5), and let-60 ras (5). Finally, we isolated seven independent cDNA clones encoding a protein (C56E6.2) with homology to small GTP binding proteins but that does not fit into a well defined class. In the final experiment we used the oligonucleotides oRB-31 and oSL1 to amplify rab family members as nonspecifically as possible. We identified clones with potential to encode proteins with similarity to rab1A (11), rab1B (1), rab2 (2), rab3 (2), rab6A (1), rab6B (1), rab7 (2), rab8 (6), rab10 (1), rab11 (13), rab14 (8), rab19A (4), rab19B (1), RAM (4), and S10 (11). The sequences have been deposited into the GenBank database (accession numbers U68250 to U68264).

Production of antibodies. The Bam HI/SspI fragment from pRB102 was inserted into pRSETB (Invitrogen, San Diego, CA) to create the plasmid pRB110. The plasmid expresses a fusion protein containing a six histidine tag on the $\mathrm{N}$ terminus of $23.5 \mathrm{kDa}$ (amino acids $6-219$ ) of the $C$. elegans RAB-3 protein. The fusion protein was purified and used to immunize mice as described in Nonet et al. (1993). Anti-RAB-3 sera were used at 1:1000 dilution. Rabbit anti-synaptotagmin (SNT-1) antisera were raised by intramuscular injection of $150 \mu \mathrm{g}$ of bacterially expressed fusion protein, described in Nonet et al. (1993), in Freund's complete adjuvant, followed by three boosts at 1 month intervals in Freund's incomplete adjuvant. Anti-SNT-1 sera were used at 1:2000 dilution.

Immunocytochemistry. Immunocytochemistry was performed as previously described (Nonet et al., 1993), except that worms occasionally were fixed in a modified Bouin's fixative $(0.75 \mathrm{ml}$ of saturated picric acid, $0.25 \mathrm{ml}$ of formalin, $0.05 \mathrm{ml}$ of glacial acetic acid, $0.25 \mathrm{ml}$ of methanol, and $0.01 \mathrm{ml}$ of $\beta$-mercaptoethanol). SNT-1 immunoreactivity was weaker than RAB-3 immunoreactivity in Bouin's fixative, but the reverse was true, when a $4 \%$ paraformaldehyde fixation was used, as described in Nonet et al. (1993).

Localization of rab-3 to chromosome II. pRB102 insert DNA was used to probe an ordered grid of yeast artificial chromosome (YAC) clones representing most of the C. elegans genome. The $r a b-3$ probe hybridized to a single YAC clone, Y53D2, physically mapped to chromosome II (Coulson et al., 1988). Cosmids C02C12 and F11G1 were shown to contain rab-3 by Southern analysis (Coulson et al., 1986). Fragments from cosmid F11G1 were cloned to create pMK1 [6.2 kb HindIII fragment inserted into pBluescript $\left.\left.\mathrm{KS}^{-}\right)\right]$and pMK4 (9.5 kb HindIII fragment inserted into pMK1). The $r a b-3:: l a c Z$ fusion plasmid pMG122 was created by inserting the $3.1 \mathrm{~kb} P p u \mathrm{MI}-X b a \mathrm{I}$ fragment from pMK4 into pPD21.28 (Fire et al., 1990). Germline transformation was used to demonstrate that pMK4 contains a functional $r a b-3$ gene (Mello et al., 1991). pMK4 DNA $(10 \mu \mathrm{g} / \mathrm{ml})$ and the pRF4 plasmid $(100 \mu \mathrm{g} / \mathrm{ml})$ containing the dominant roller marker rol-6(su1006) were co-injected into $r a b-3(y 250)$ and $r a b-3(y 251)$. Stably transformed y250 and y251 animals were unable to grow on plates containing $0.8 \mathrm{~mm}$ aldicarb, in contrast to their untransformed siblings. F2 animals bearing the extrachromosomal sequences contained elevated levels of RAB-3 product as detected with the RAB-3 antisera (data not shown).

Isolation of rab-3 mutants. Wild-type males were mutagenized for $4 \mathrm{hr}$ in $50 \mathrm{~mm}$ ethyl methanesulfonate (EMS). Males were crossed en masse to dpy-25(e817)/ccDf5 hermaphrodites. The crosses were transferred twice daily to new plates, and aldicarb (100 mM stock in ethanol) was added to $0.8 \mathrm{~mm}$ on plates containing progeny. Seventy-five independent aldicarbresistant strains were isolated from an estimated 150,000 cross progeny. Each potential rab-3 mutation was made homozygous by identifying animals which failed to segregate dead eggs. Of the 75 strains, 19 showed a clearly visible uncoordinated phenotype. All Unc strains were tested to determine whether they were likely to harbor $r a b-3$ lesions by crossing mutant/+ males into $c c D f 5 / d p y-25$ animals. In no case were Unc cross progeny observed, suggesting that all lesions conferring the Unc phenotype lay outside the boundaries of $c c D f 5$. Animals from the other 56 strains were fixed for immunohistochemistry and examined with antiRAB-3 antibodies to identify mutants. Both the immunohistochemical defect and aldicarb resistance of $y 250$ and $y 251$ were linked to rol-6, suggesting they were $r a b-3$ lesions, whereas defects in $y 255$ animals were unlinked to rol-6. y255 was demonstrated to be an allele of aex-3 (Iwasaki et al., 1997). $r a b-3$ was positioned between bli-2 and $d p y-10$ by three- 


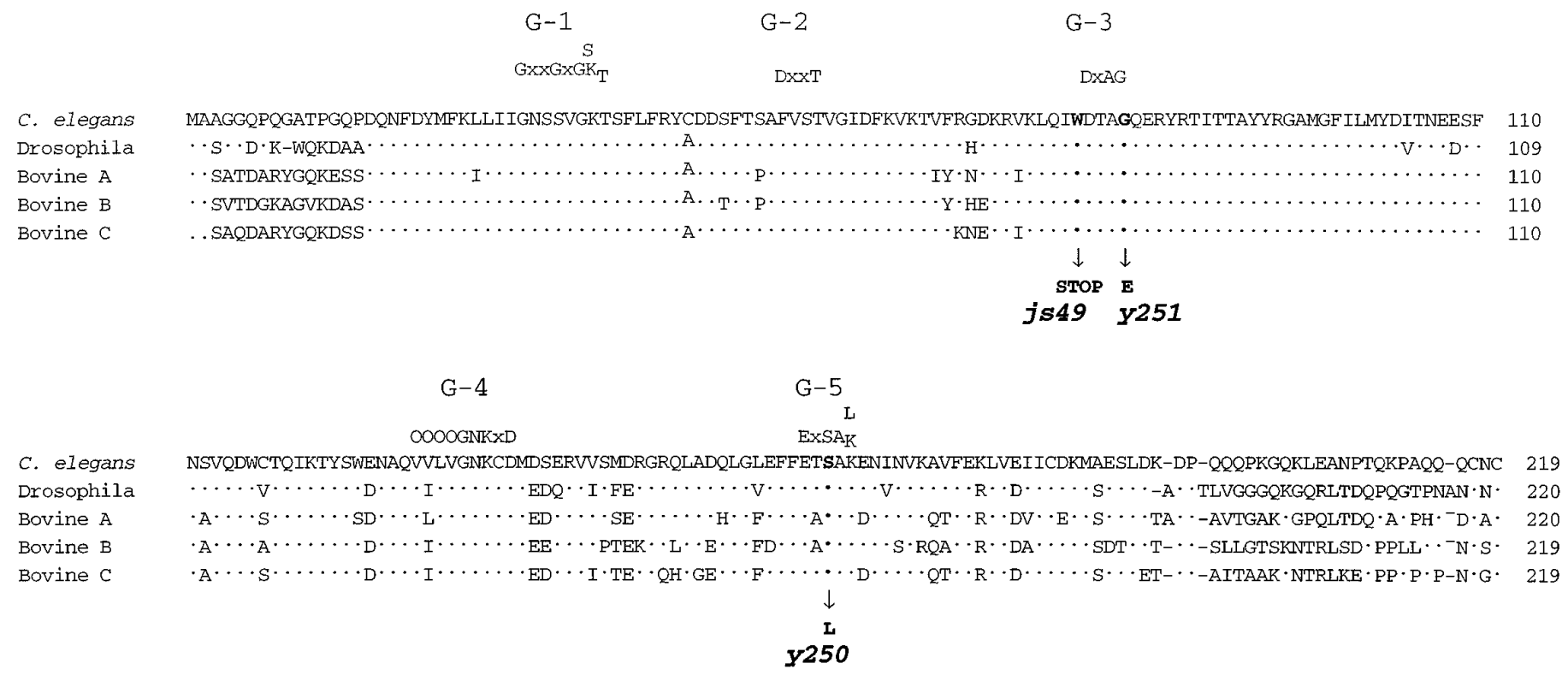

Figure 1. Similarity among rab3 proteins from metazoa. Alignment of C. elegans, Drosophila, and bovine rab3 proteins. The locations of five conserved domains involved in the binding of guanine nucleotides are labeled $G-1$ to $G-5$ (Bourne et al., 1991). The $C$. elegans protein shares $76 \%$ identity with Drosophila rab3, 73\% identity with both bovine rab3A and rab3C, and 71\% identity to both bovine rab3B and murine rab3D. The positions of amino acid substitutions or stop codons identified in $r a b-3$ mutants are indicated. $j 449$ is a $\mathrm{G}$ to $\mathrm{A}$ transition at position 2 of codon $76, y 250$ is a $\mathrm{C}$ to $\mathrm{T}$ transition at position 2 of codon 165, and y251 is a G to A transition at position 2 of codon 80. Dots represent identity among all proteins. The standard single amino acid code is used. $O$ represents a hydrophobic amino acid, and $X$ represents any amino acid. Amino acid numbering appears on the right.

factor mapping. From rab-3/bli-2(st1016) dpy-10(e128), Bli non-Dpy animals were isolated. None of 17 Bli non-Dpy animals carried $r a b-3(y 251)$ as assayed by aldicarb resistance, immunohistochemistry, and PCR analysis. Of the Bli non-Dpy recombinants, 1 of 16 contained the $r a b(y 250)$ lesion as assayed by aldicarb resistance and immunohistochemistry. $r a b$ $3(j s 48)$ and $r a b-3(j 49)$ were isolated in a noncomplementation screen Wild-type males were mutagenized with EMS as described above and crossed to rab-3(y250) bli-2(st1016) II; xol-1(y9) flu-2(e1003) $X$ and adult cross progeny assayed for aldicarb resistance. All $r a b-3$ lesions were characterized molecularly by direct DNA sequencing of PCR products (Sequenase, United States Biochemicals, Cleveland, $\mathrm{OH}$ ) amplified from genomic DNA isolated from homozygous mutant animals. The entire coding region and all intron/exon boundaries were sequenced in all cases.

Behavioral assays. Chemotaxis assays on populations were performed essentially as previously described (Bargmann et al., 1993). Indexes presented in Table 1 are calculated from six individual assays. Defecation was observed under a dissecting microscope, and cycles were recorded with a simple computer program (Liu and Thomas, 1994). Data shown represent observations of at least five animals for $>10 \mathrm{~min}$. Pharyngeal pumping was assayed by counting pumping for five $1 \mathrm{~min}$ intervals. Data shown represent the mean of at least eight animals for each genotype. Mating assays were done as previously described (Hodgkin, 1983). Data shown represent the mean of four assays.

Resistance to aldicarb. Mutants were assayed for responses to both chronic and acute exposure to aldicarb. For chronic resistance, five young gravid adults were placed on plates containing aldicarb. Worms are considered resistant to chronic exposure if the animals are capable of exhausting the E. coli food supply from the plates within $10-12 \mathrm{~d}$ at $20^{\circ} \mathrm{C}$. Acute aldicarb resistance was examined by transferring individual animals to plates containing aldicarb and assaying for paralysis $5 \mathrm{hr}$ after exposure. Animals were considered paralyzed if they failed to move even if prodded with a platinum wire.

Acetylcholine biochemistry. Acetylcholine levels were measured essentially as described in Nonet et al. (1993), using the methods of McCaman and Stetzler (1977).

Electrophysiology. Electropharyngeograms (EPGs) were recorded with a GRASS P15 amplifier and LabView Acquisition software as previously described (Avery et al., 1995). Bath solution consisted of Dent's saline with $2 \mathrm{~mm}$ serotonin to stimulate pumping. Recordings used for analysis were taken from preparations that showed strong signal-to-noise ratios. The presence of MC and M3 transients were scored qualitatively as distinct spikes greater than the background noise. Statistical analysis was performed on EPGs collected from ten sequential $5 \mathrm{sec}$ traces for each worm. Data presented represent the mean of at least eight animals per strain.

Electron microscopy. Worms were cut in $0.8 \%$ glutaraldehyde and $0.7 \%$ osmium tetroxide in $0.1 \mathrm{M}$ cacodylate, $\mathrm{pH} 7.4$, on ice. After $2 \mathrm{hr}$ they were moved to $2 \%$ osmium tetroxide in $0.1 \mathrm{~m}$ cacodylate, $\mathrm{pH} 7.4$, and left at $4^{\circ} \mathrm{C}$ overnight. Processing and sectioning were as previously described (McIntire et al., 1992).

\section{RESULTS}

We used PCR to identify sequences encoding proteins with similarity to the GTPase rab3 in $C$. elegans. We obtained cDNAs representing the two alternative transcripts of the gene. The smaller transcript contained a 219 amino acid open reading frame with $76 \%$ identity to Drosophila rab3 (Fig. 1). This conservation of sequence is restricted to the central 190 amino acid core of the protein, which retains over $88 \%$ identity with Drosophila rab3. By contrast, the $\mathrm{N}$ - and $\mathrm{C}$-terminal regions of $C$. elegans rab3 diverge significantly from both Drosophila and vertebrate rab3 members, except for the presumptive CXC prenylation site at the $\mathrm{C}$ terminus (Johnston et al., 1991). The gene was named rab-3 in accordance with C. elegans nomenclature guidelines (Horvitz et al., 1979).

A longer transcript derived from the $r a b-3$ locus contains an additional exon with the potential to encode a larger RAB-3 isoform extended by 14 amino acids (MNNQQAAIASARSR) on the $\mathrm{N}$ terminus. The additional exon was present in 2 of $15 \mathrm{cDNAs}$ we isolated, and a correspondingly larger transcript was visible on Northern blots (data not shown). Transcription of this RNA is likely to initiate from an independent promoter, because a genomic clone lacking this additional exon rescues $r a b-3$ mutants (see below). The additional exon resides $3 \mathrm{~kb}$ upstream of the more common first exon. Similarly, an additional exon encoding identical residues at 13 of 14 codons (MNNQQAAIASARNR) was also present in the $C$. briggsae $r a b-3$ gene $3 \mathrm{~kb}$ upstream of the more 

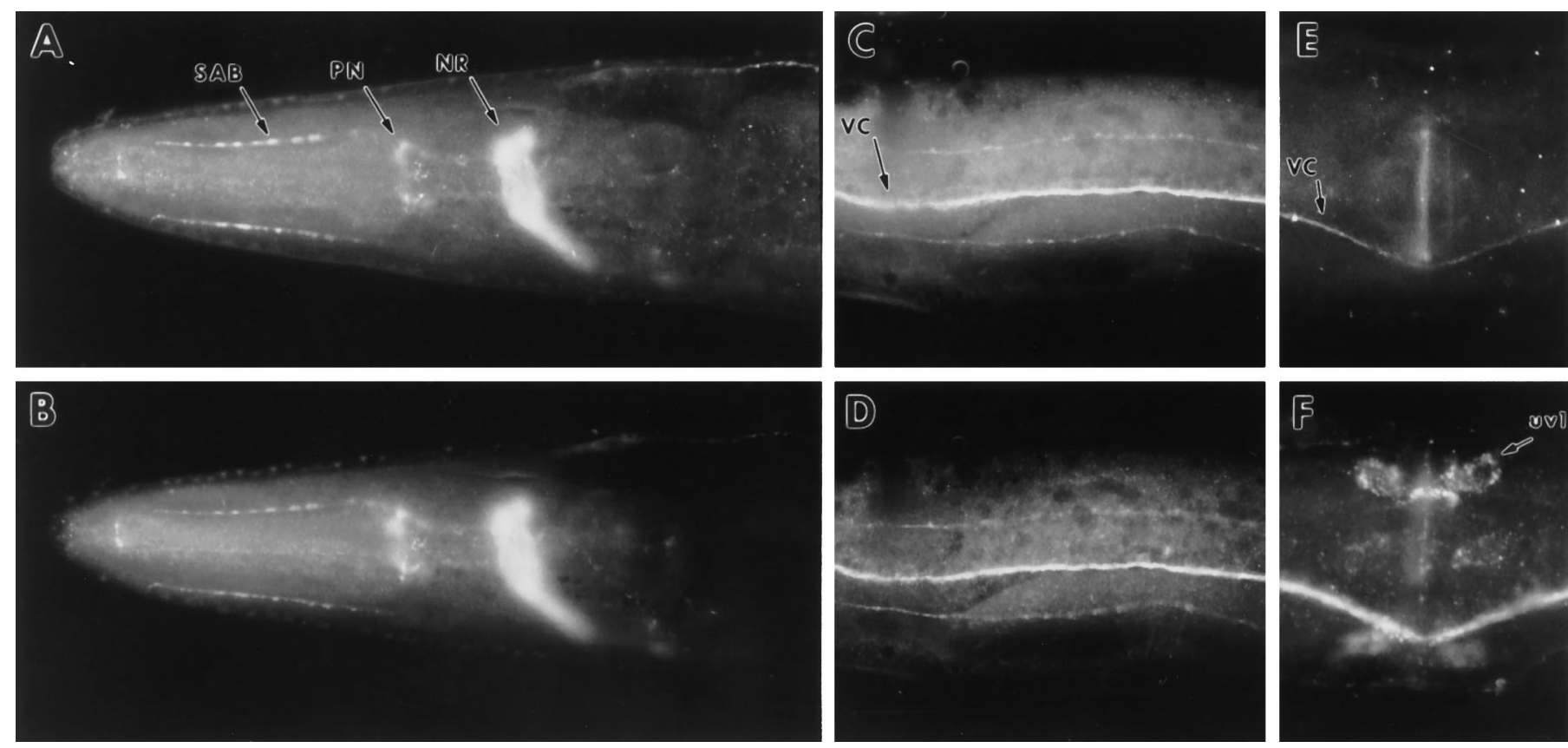

Figure 2. RAB-3 expression in the C. elegans nervous system. Whole worms were fixed and stained with anti-RAB-3 and anti-synaptotagmin primary antibodies and visualized with FITC- or Cy3-conjugated antibodies. $A, B$, Lateral view of the head region of a wild-type adult hermaphrodite showing RAB-3 $(A)$ and synaptotagmin $(B)$ immunoreactivity in the nerve ring $(N R)$, pharyngeal nervous system ( $P N)$, and $S A B$ neuron axonal processes (arrows). $C, D$, Ventral view of the midsection of an adult hermaphrodite showing RAB-3 $(C)$ and synaptotagmin $(D)$ immunoreactivity in the ventral nerve cord $(V C$; arrow) and the ventral sublateral processes. $E, F$, Lateral view of the vulval region of an adult hermaphrodite illustrating the absence of RAB-3 $(E)$ but the presence of synaptotagmin $(F)$ immunoreactivity in the uv1 cells of the somatic gonad (uv1; arrow). The ventral cord is also visible (VC).

common first exon. These species are morphologically similar but genetically divergent; they are about as divergent at the nucleotide level as mouse is from human (Fitch et al., 1995). The conservation of this $r a b-3$ sequence in the two nematode species suggests that this alternative exon is functional. We have not examined the role of this N-terminal sequence in C. elegans.

\section{Search for additional C. elegans rab genes failed to identify RAB-3 isoforms}

We searched for other $r a b-3$ homologs in the C. elegans genome to determine whether other isoforms were present in C. elegans, as is the case in vertebrates (Fischer von Mollard et al., 1990; Mizoguchi et al., 1990). Because we were unable to identify sequences that encode molecules similar to $r a b-3$ by low-stringency hybridization (data not shown) or by analysis of available C. elegans genomic and EST sequence data, we resorted to PCR-based methods. We performed three PCR experiments (details are discussed in Materials and Methods) capable of amplifying both $r a b-3$ and more divergent $r a b$ family members, reasoning that if we isolated clones encoding less conserved members of the family we also should retrieve clones encoding novel conserved $r a b-3$ family members. We isolated both the $C$. elegans $r a b-3$ gene ( $>100$ times) and many additional $r a b$ family members from cDNA (details are discussed in Materials and Methods). In summary, we isolated and characterized clones encoding portions of $16 r a b$ and more distant ras super-family members. Our search for $r a b$ family members isolated all $C$. elegans $r a b$ family members present in sequence databases except a gene homologous to rab5, a $r a b$ member that is relatively divergent from $r a b 3$. However, despite identifying several novel genes encoding divergent $r a b$ molecules, we failed to identify additional rab3 genes from $C$. elegans.

\section{C. elegans RAB-3 protein is expressed in the nervous system}

We raised antisera against a $\mathrm{RAB}-3$ fusion protein produced in $E$. coli to examine the distribution of the protein in $C$. elegans. Antisera were used to stain whole mounts of $C$. elegans. Immunoreactivity was detected in the synaptic-rich regions of the nervous system in a pattern similar to the distribution of the $C$. elegans synaptic vesicle membrane protein synaptotagmin (Fig. 2) (Nonet et al., 1993). Specifically, the nerve ring (Fig. $2 A, B$ ), ventral nerve cord (Fig. 2C,D), and dorsal nerve cord (data not shown) showed the highest immunoreactivity. $r a b-3$ expression also was observed in the pharyngeal nervous system (Fig. $2 A, B$ ). Detectable levels of RAB-3 were rarely observed in neuronal cell bodies, dendrites of sensory neurons, or the commissural tracts of motor neurons. In contrast to synaptotagmin, RAB-3 protein was not observed at significant levels in the gonadal uv1 neurosecretory cells (Fig. 2E,F). Additionally, RAB-3 was not observed in muscle, hypodermis, or intestinal tissue. Because RAB-3 protein was not detectable in neuronal cell bodies, we fused the coding sequence of the reporter gene lac $Z$ containing a nuclear localization signal to $r a b-3$ coding sequences to determine in which neurons the gene was expressed. Analysis of transgenic animals carrying the $r a b-3:: l a c Z$ reporter construct pMG122 confirmed that the gene is expressed in most, if not all, neurons (data not shown) but is not expressed in other tissues. Thus, both lac $Z$ reporter constructs and immunohistochemistry confirmed that $r a b-3$ expression was restricted to neuronal tissues, as previously has been observed for many rab3 genes in vertebrates (Fischer von Mollard et al., 1990; Mizoguchi et al., 1990). 

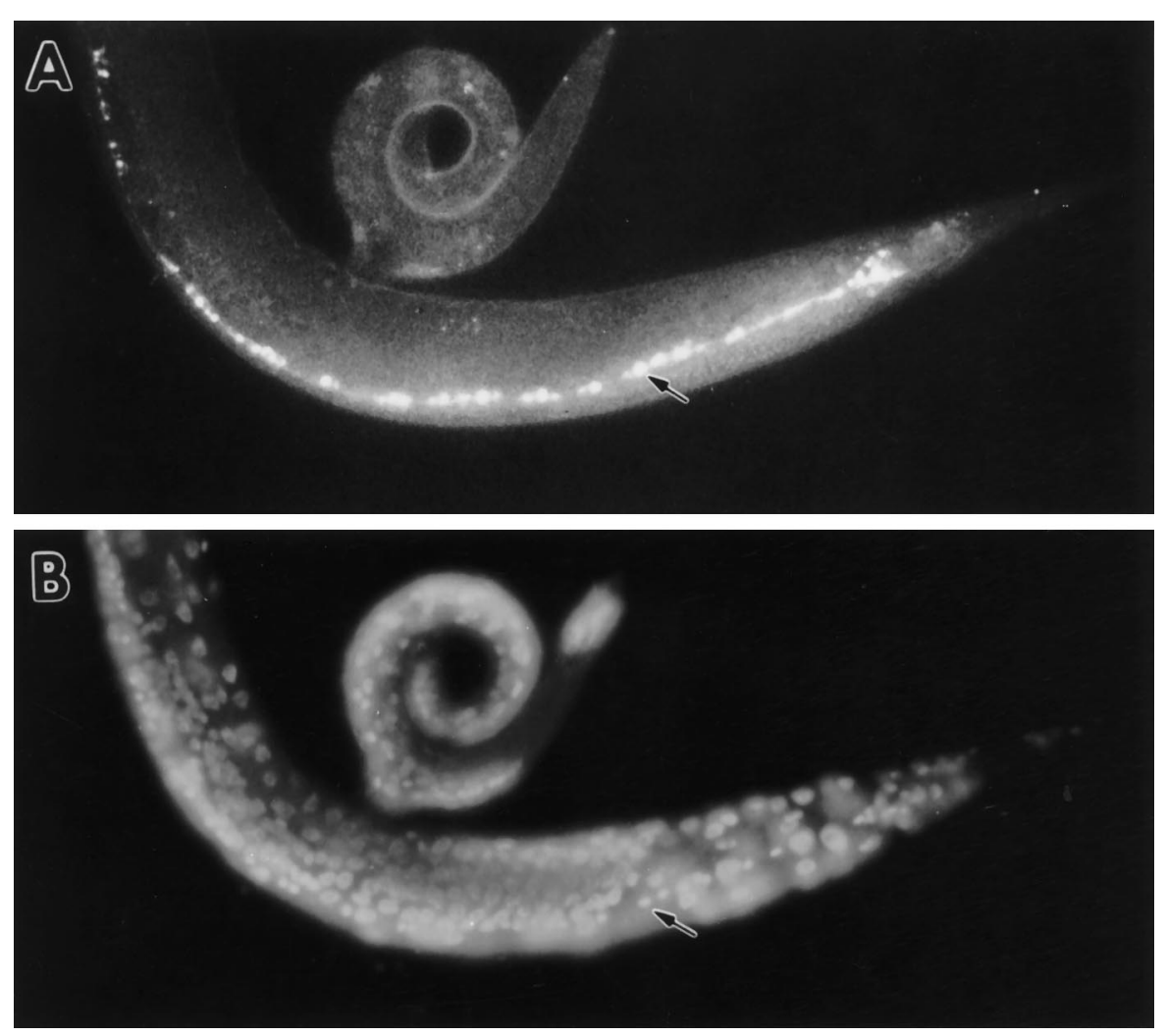

Figure 3. Mislocalization of RAB-3 in unc-104 mutants. Whole unc-104(e1265) nematodes were fixed and stained with anti-RAB-3 primary antibodies and visualized with FITC-conjugated secondary antibodies. The anterior of the animal is to the left. $A$, Oblique lateral view of a young adult hermaphrodite showing RAB-3 immunoreactivity localized to neuronal cell bodies in the ventral nerve cord (arrow). B, DAPI staining of nuclei of the animal shown in $A$. A row of neuronal nuclei located in the ventral nerve cord is visible (arrow).

\section{RAB-3 colocalizes with synaptic vesicles}

The vertebrate rab3A and rab3C molecules associate specifically with synaptic vesicles (Fischer von Mollard et al., 1990, 1994a). Similarly, C. elegans RAB-3 colocalized with synaptic vesicles. First, RAB-3 immunoreactivity colocalized with the synaptic vesicle protein synaptotagmin (see Fig. 2). Second, when synaptic vesicles were mislocalized, RAB-3 immunoreactivity similarly was mislocalized. Specifically, in unc-104 mutants, synaptic vesicles accumulated to high levels in neuronal cell bodies and so did RAB-3 immunoreactivity (Fig. 3). unc-104 encodes a kinesin-like molecule that is required for the transport of synaptic vesicles from cell bodies to synapses in C. elegans (Hall and Hedgecock, 1991). Vertebrate homologs of $u n c-104$ similarly are required for transport of synaptic vesicles proteins, but not other neuronal components (Okada et al., 1995). The synaptic vesicle protein synaptotagmin (Nonet et al., 1993) also accumulates in cell bodies in unc-104 mutants, but the plasma membrane protein syntaxin remains in processes (M. L. Nonet, unpublished data). We concluded that the $C$. elegans RAB-3 molecule is vesicle-associated, as has been demonstrated biochemically for vertebrate rab3 proteins.

\section{Isolation of rab-3 mutants}

The $r a b$-3 gene was positioned near bli-2 on chromosome II, using a combination of molecular and genetic methods (Fig. 4). We used the deficiency $c c D f 5$, which removes a region spanning the $r a b-3$ gene, to isolate $r a b-3$ mutations. We reasoned that certain mutations in the $r a b-3$ gene would result in a reduced ability to secrete acetylcholine at neuromuscular synapses. To identify these secretion-defective mutants, we screened for animals resistant to the acetylcholinesterase inhibitor aldicarb, because mutations in genes encoding other $C$. elegans synaptic components all confer aldicarb resistance (Nonet et al., 1993; Nguyen, 1995). We isolated mutations that conferred an aldicarb-resistant phenotype in trans to $c c D f 5$ (details are discussed in Materials and Methods) and identified $y 250$ and $y 251$ as $r a b-3$ mutants because they displayed altered RAB-3 immunoreactivity (see below). An additional allele, js49, subsequently was isolated in a noncomplementation screen, using aldicarb selection to identify candidate mutants (see Materials and Methods). y250 and y251 mutations result in missense changes in amino acids that are thought to be essential for guanine nucleotide binding and are conserved among all small monomeric GTP-binding proteins (Fig. 1). js49 is a nonsense mutation in the tryptophan 76 codon (Fig. 1).

Genetic and immunohistochemical data suggested that all three alleles are complete or almost complete loss-of-function mutations. First, for each allele, the homozygous mutant phenotype is similar to the phenotype caused by the allele in trans to the deficiency $c c D f 5$ (data not shown). Second, RAB-3 protein was not detected in $y 250$ or js 49 mutants, using RAB-3 antibodies in whole-mount immunocytochemistry. The residual RAB-3 immunoreactivity in y251 animals was mislocalized to all neuronal cell bodies (Fig. 5). The lack of RAB-3 immunoreactivity was not caused by improper synaptic terminal development, because the synaptic components synaptotagmin (Fig. 5), synaptobrevin, and syntaxin (data not shown) were normally expressed and localized in all three mutants. Using anti-synaptotagmin antisera, we could not detect a difference in the appearance or number of synaptic varicosities in the SAB neurons that innervate head muscle between $r a b-3(j s 49)(12 \pm 2.3, n=31)$ and the wild type (11.6 \pm 2.3 , $n=31$ ). Additionally, we examined all axonal tracts, using antisyntaxin antibodies (M. L. Nonet, unpublished data), and confirmed that they were positioned normally in all three mutants (data not shown). Together, these data suggested that the three rab-3 mutants do not retain any significant RAB-3 activity and 
A

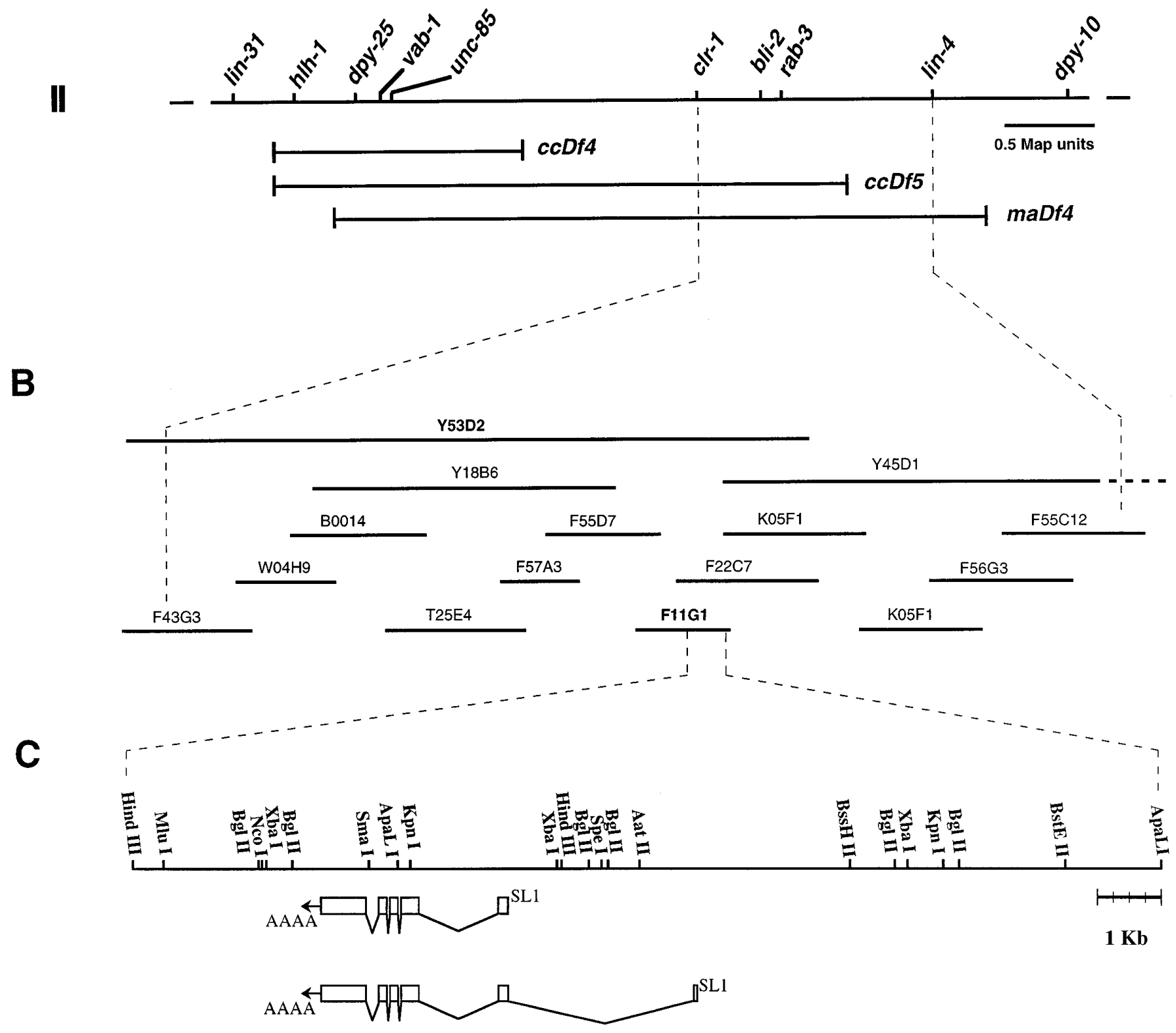

\section{pMK1}

\section{pMK4}

\section{pMG122}

Figure 4. The rab-3 locus. A, Genetic map of a portion of chromosome II, illustrating the position of genes and deficiencies used for the mapping and isolation of $r a b-3$ mutants. $B$, Organization of the physical region neighboring $r a b$-3. A series of overlapping yeast artificial chromosome and cosmid clones surrounding the $r a b-3$ gene is shown. The positions of the $c l r-1$ and lin-4 genes are delineated on the physical map. rab-3 was positioned in this interval as a result of specific hybridization to the YAC and cosmid clones (in bold). $C$, Restriction map of a portion of cosmid F11G1. Exons of the two transcripts derived from $r a b-3$ are illustrated. The trans-spliced leader SL1 is found at the 5' end of both $r a b-3$ messages. SL1 and $A A A A$ mark the trans-spliced leader attachment and poly $\left(\mathrm{A}^{+}\right)$addition sites, respectively. The genomic inserts of plasmid clones used in our experiments are illustrated as solid lines. pMG122 is a translational lac $Z$ fusion to the $r a b-3$ coding sequence at amino acid 194. 

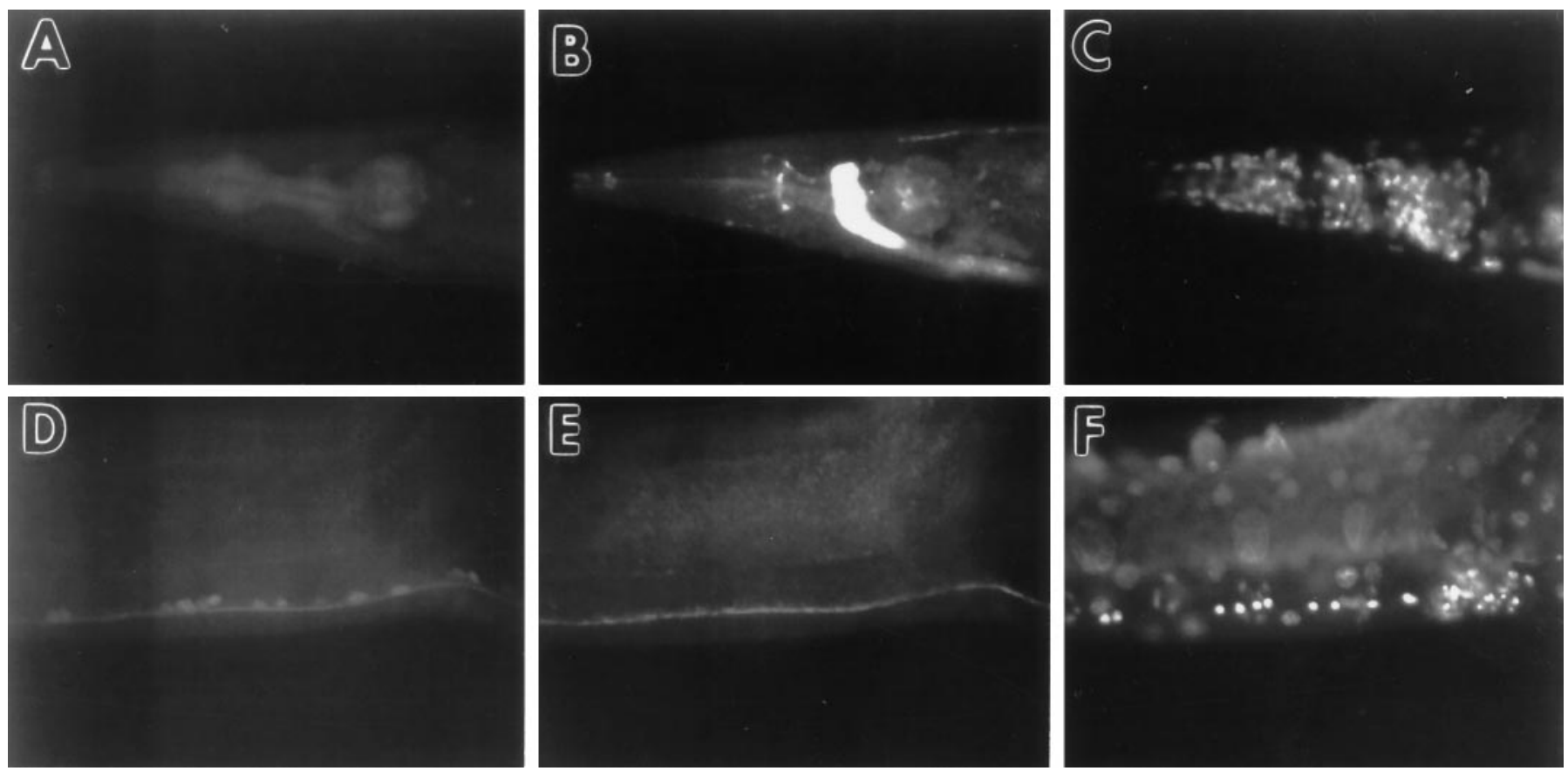

Figure 5. RAB-3 protein is absent or mislocalized in $r a b-3$ mutants. Whole worms were fixed and stained with primary antibodies and visualized with FITC-or cy3-conjugated antibodies. $A-C$, Lateral view of the head region of a $r a b-3(j s 49)$ adult stained with anti-RAB-3 antibodies $(A)$, antisynaptotagmin antibodies $(B)$, and DAPI to visualize nuclei $(C) . D-F$, Ventral view of the midsection of $r a b-3(y 251)$ hermaphrodite stained with anti-RAB-3 antibodies $(D)$, anti-synaptotagmin antibodies $(E)$, and DAPI to visualize nuclei $(F)$.

Table 1. Analysis of behaviors of $r a b-3$ mutants

\begin{tabular}{|c|c|c|c|c|c|}
\hline Strain & Chemotaxis index ${ }^{a}$ & Pumping rate ${ }^{b}$ & Defecation cycle period $^{c}$ & Mating efficiency $(\%)^{d}$ & ACh levels ${ }^{e}$ \\
\hline Wild type (N2) & $0.86 \pm 0.04$ & $250 \pm 30$ & $45 \pm 3.5$ & 100 & $0.35 \pm 0.02$ \\
\hline$r a b-3(y 250)$ & $0.69 \pm 0.05$ & $173 \pm 13$ & $59 \pm 9.3$ & 9 & $0.44 \pm 0.06$ \\
\hline$r a b-3(y 251)$ & $0.72 \pm 0.06$ & $158 \pm 15$ & $58 \pm 8.0$ & 24 & $0.35 \pm 0.08$ \\
\hline$r a b-3(j s 49)$ & N.D. & $157 \pm 10$ & $56 \pm 4.3$ & 35 & N.D. \\
\hline snt $-1(m d 290)^{*}$ & $0.01 \pm 0.02$ & $60.3 \pm 30$ & $141 \pm 66$ & 0 & $2.07 \pm 0.24$ \\
\hline
\end{tabular}

\section{Means are presented \pm SD. N.D., Not determined.}

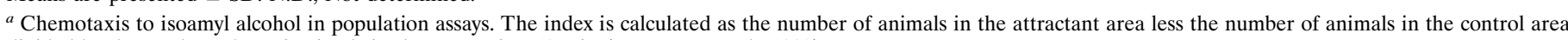
divided by the total number of animals in the assay after 60 min (Bargmann et al., 1993).

${ }^{b}$ Pharyngeal pumps per minute on food.

${ }^{c}$ Seconds between defecation cycles.

${ }^{d}$ Efficiency of mating to $d p y-11(e 224)$ animals as a percentage of wild-type efficiency.

${ }^{e}$ Acetylcholine levels in picomoles per microgram of protein.

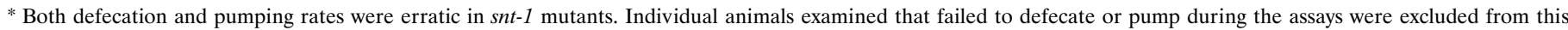
analysis. Such animals represent approximately one-third of snt-1 animals.

that the absence of $r a b-3$ activity does not lead to abnormalities in synaptic development.

\section{rab-3 mutants have very mild behavioral defects}

rab-3 mutants exhibited mild behavioral defects. The three rab-3 mutants $y 250, y 251$, and $j 449$ were indistinguishable at a behavioral level. With careful observation, $r a b-3$ mutant animals often could be distinguished from wild-type animals on the basis of the increased amplitude (loopy) and slower speed of their sinusoidal locomotion. In assays that quantified chemotaxis toward the volatile odorant isoamyl alcohol (Bargmann et al., 1993), rab-3 animals were distinguishable from wild-type animals but remained fairly effective at this task, as compared with more severe synaptic mutants like snt-1(md290) animals that lack synaptotagmin (Table 1). Other behaviors, including pharyngeal pumping and defecation, were also slightly abnormal (Table 1). Neverthe- less, $r a b-3$ males animals were capable of mating, although at a lower efficiency than wild-type animals (Table 1). Finally, the aldicarb-resistant phenotype of all three mutants was recessive, suggesting that none of the mutations has significant gain-offunction or dominant negative characters. Although these abnormalities were subtle, $r a b-3$ animals still displayed several deficiencies in synaptic function and organization.

\section{rab-3 mutants are resistant to an inhibitor of acetylcholinesterase}

To assess the synaptic transmission deficits of rab-3 mutants, we first quantified the resistance of $r a b-3$ mutants to the acetylcholinesterase inhibitor aldicarb. Although $r a b-3$ mutants were more resistant to aldicarb than wild-type animals (Fig. 6), this resistance was substantially weaker than the resistance of animals carrying mutations in other genes encoding synaptic components 


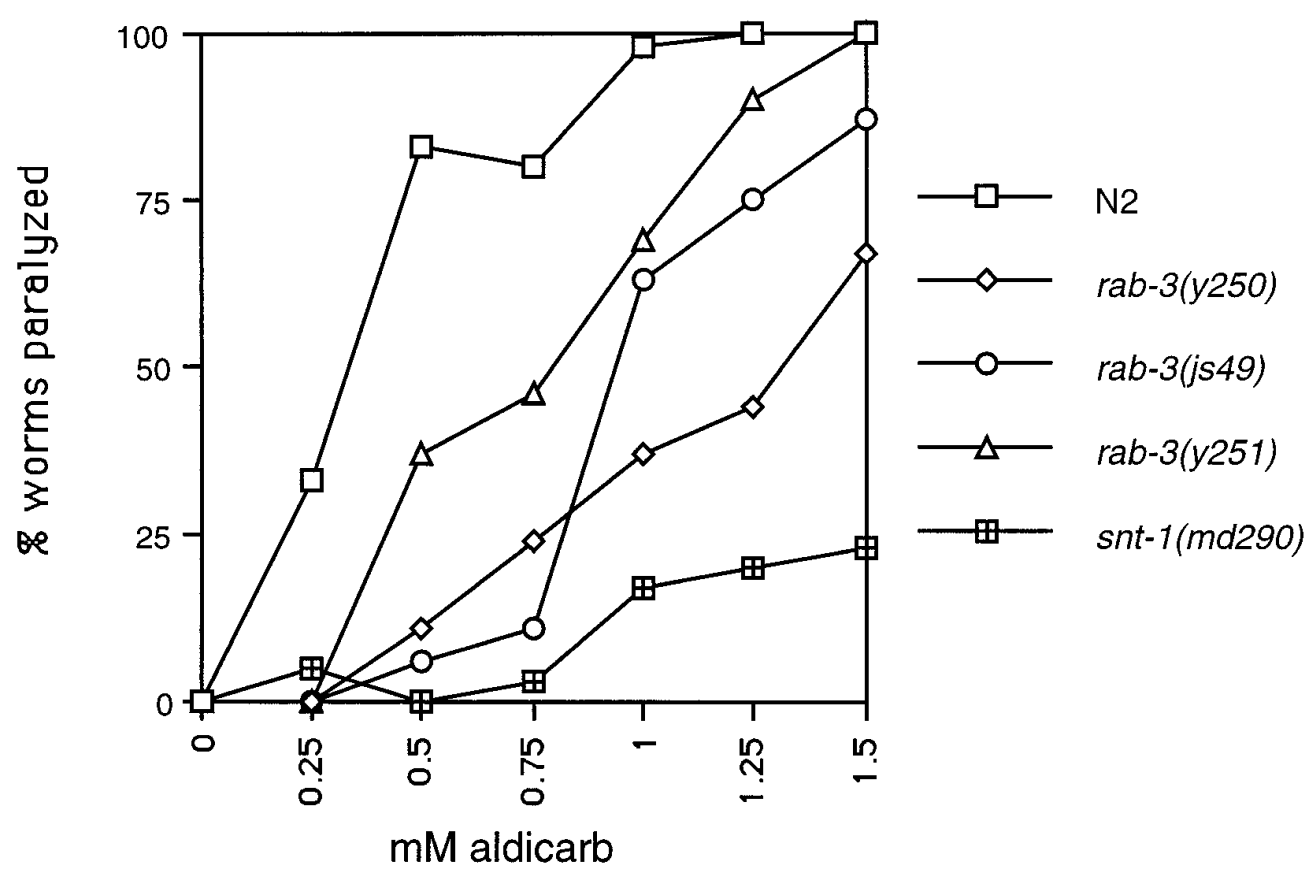

Figure 6. rab-3 mutants are resistant to an inhibitor of acetylcholinesterase. Young adults worms were assayed for acute body paralysis after a $5 \mathrm{hr}$ incubation with aldicarb on agar plates containing food. Twenty to twenty-five animals were assayed at each concentration.

Table 2. Analysis of electropharyngeogram transients in rab-3 mutants

\begin{tabular}{|c|c|c|c|c|}
\hline & Wild-type & js49 & $y 250$ & $y 251$ \\
\hline M3s/pump & $2.5 \pm 0.6$ & $1.2 \pm 0.3$ & $0.5 \pm 0.3$ & $1.0 \pm 0.4$ \\
\hline Pump duration (msec) & $123 \pm 7$ & $160 \pm 17$ & $164 \pm 12$ & $140 \pm 19$ \\
\hline Subthreshold MCs/pump & 0.7 & 5.5 & 1.1 & 2.1 \\
\hline Nonsynchronous MCs/total subthreshold MCs & 0.05 & 0.51 & 0.59 & 0.67 \\
\hline
\end{tabular}

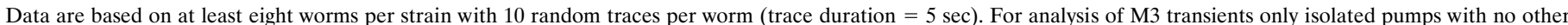

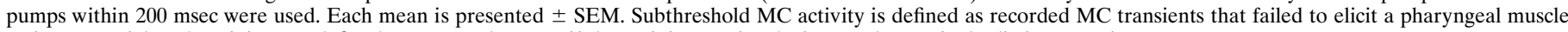
action potential. MC activity was defined as nonsynchronous if the activity consisted of more than a single distinct transient.

such as synaptotagmin (Nonet et al., 1993; Nguyen, 1995). We also examined the levels of the neurotransmitter acetylcholine in the $r a b$ mutants, because other mutants lacking synaptic components accumulate this transmitter (Hosono et al., 1987; Hosono and Kamiya, 1991; Nonet et al., 1993; Nguyen, 1995). However, acetylcholine levels were normal in rab-3 mutants (Table 1). This was not unexpected because the increase in ACh levels in synaptic transmission mutants is only modest (four- to fivefold) even in the most severe transmission mutants (Hosono et al., 1987; Nguyen, 1995). rab-3 mutants also responded normally to the acetylcholine receptor agonist levamisole (Lewis et al., 1980), suggesting that postsynaptic organization was qualitatively normal (data not shown). Together, these data indicated that transmitter release from cholinergic neurons is generally decreased in rab-3 mutants.

\section{rab-3 mutant synapses exhibit impaired activity}

Synaptic and endogenous muscle currents can be measured from the $C$. elegans pharyngeal muscle by using an extracellular probe. Such recordings are called electropharyngeograms or EPGs (Raizen and Avery, 1994). The motor neurons M3 and MC produce hyperpolarizing and depolarizing currents, respectively, in the pharyngeal muscle (Avery, 1993; Raizen et al., 1995). The M3 motor neuron induces repolarization of pharyngeal muscle and shortens the pump duration. $r a b-3$ mutants exhibited longer pump durations than the wild type, suggesting a decrease in M3 function (Table 2). M3 synaptic currents in wild-type worms were large in amplitude and formed coherent transients (circles, Fig. 7A). M3 transients in rab-3 mutants were less frequent, as compared with the wild type $(p \leq 0.05)$; they were smaller in amplitude (Fig. 7B, C, Table 2) and were less synchronous.

Raizen et al. (1995) have shown that activity of the MC neuron stimulates pharyngeal pumping. Isolated MC transients can be observed in wild-type animals, and these are presumably subthreshold transients that fail to elicit a pump. The frequency of subthreshold MC transients relative to successful pumps increased in rab-3 mutants (Table 2). Moreover, these subthreshold MC transients in the $r a b-3$ mutants were decreased in amplitude and were less synchronous (bursting phenotype; Fig. 7D,E). These data suggest that the MC neuron in the $r a b-3$ mutant is active but has a decreased efficacy.

\section{Depletion of synaptic vesicles at terminals in rab-3 mutants}

To characterize further the synaptic defects in $r a b-3$ mutants, we examined synaptic terminals of mutants at the ultrastructural level. We examined ventral nerve cord neuromuscular junctions, which are formed by both excitatory cholinergic and inhibitory GABAergic motor neurons. C. elegans neuromuscular junctions are distinguished by an electron-dense presynaptic density (Fig. $8)$. We quantified the number of vesicles in sections containing a presynaptic density (Table 3 ). The terminals of rab-3 mutants contained a two- to threefold reduction of synaptic vesicles, as compared with the terminals of wild-type animals. This depletion 
A) wild type

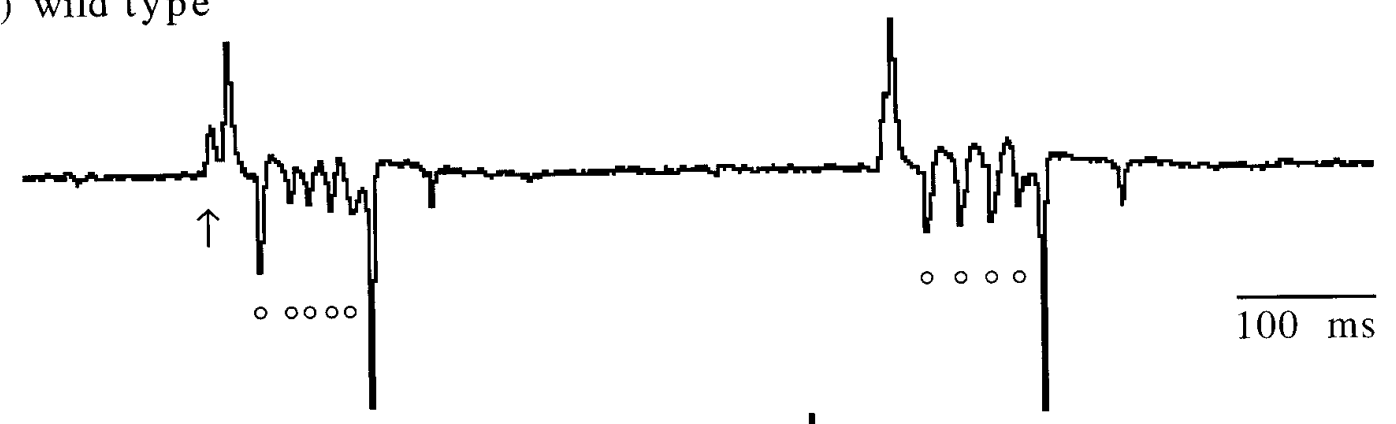

B) $r a b-3(j s 49)$

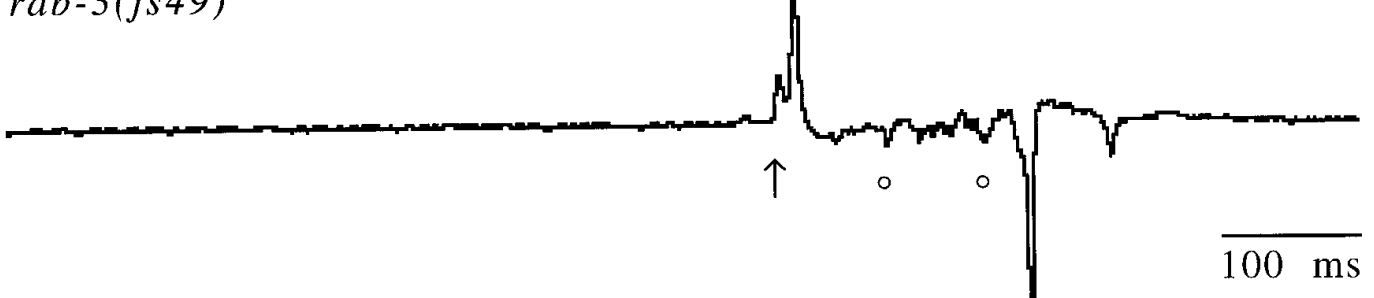

C) $r a b-3(y 250)$

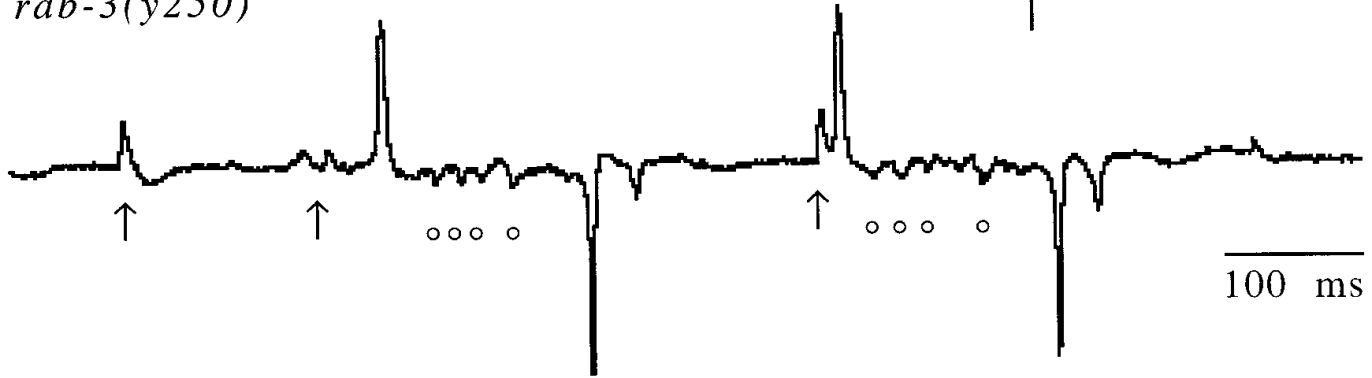

D) wild type
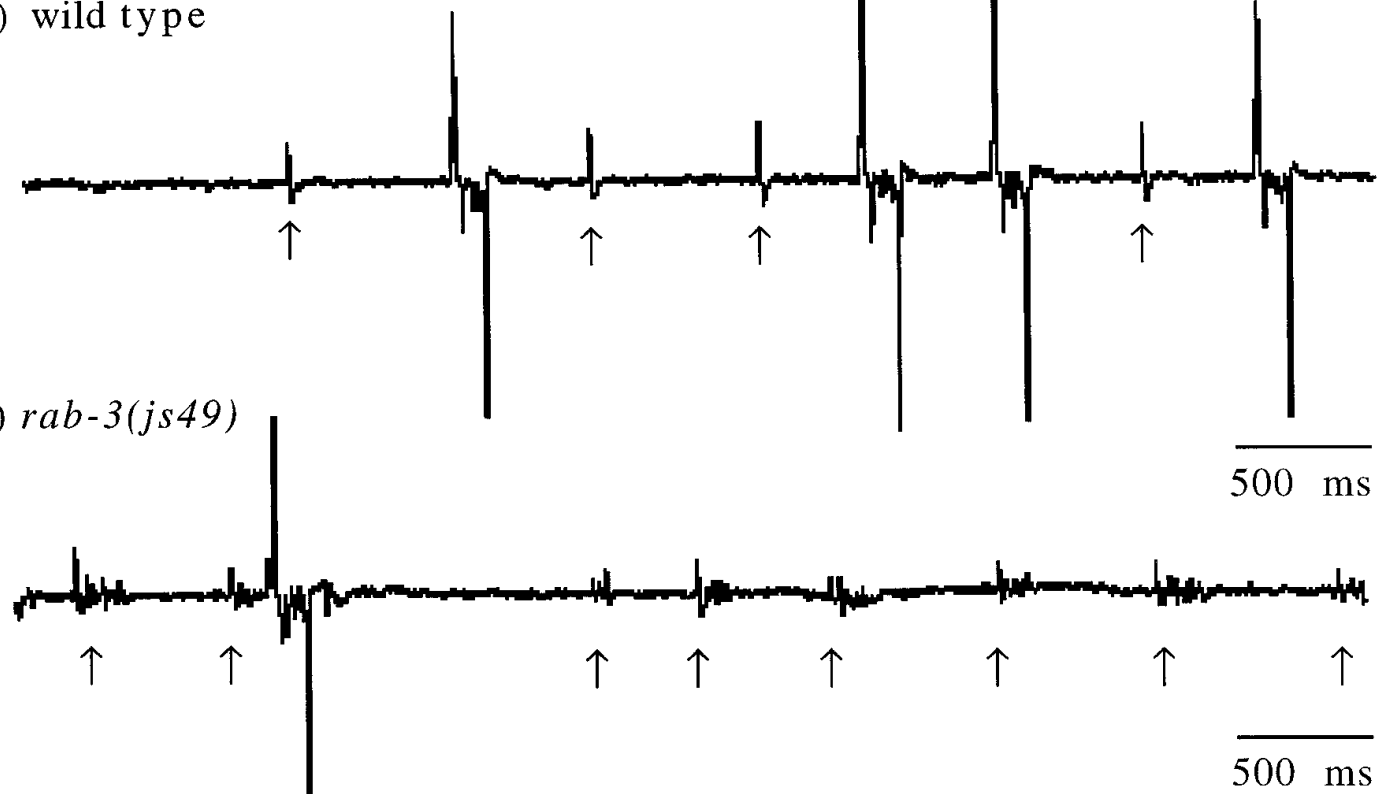

Figure 7. Pharyngeal recordings from wild-type and $r a b-3$ mutants. Characteristic recordings of a $(A, D)$ wild-type worm, $r a b-3(j s 49)(B, E)$, and $r a b-3(y 250)(C)$ mutants. Arrows indicate the MC-induced transients, and the circles indicate M3-induced transients. In wild-type animals $(D)$, MC activity that failed to initiate a pharyngeal pump was characteristically observed as a single transient. However, in $r a b-3$ mutants $(E)$, MC activity that failed to initiate a pharyngeal pump was nonsynchronous, typically consisting of a small burst of transients spaced in a 50-100 msec interval. All traces are $\mathrm{mV}$ versus time. 

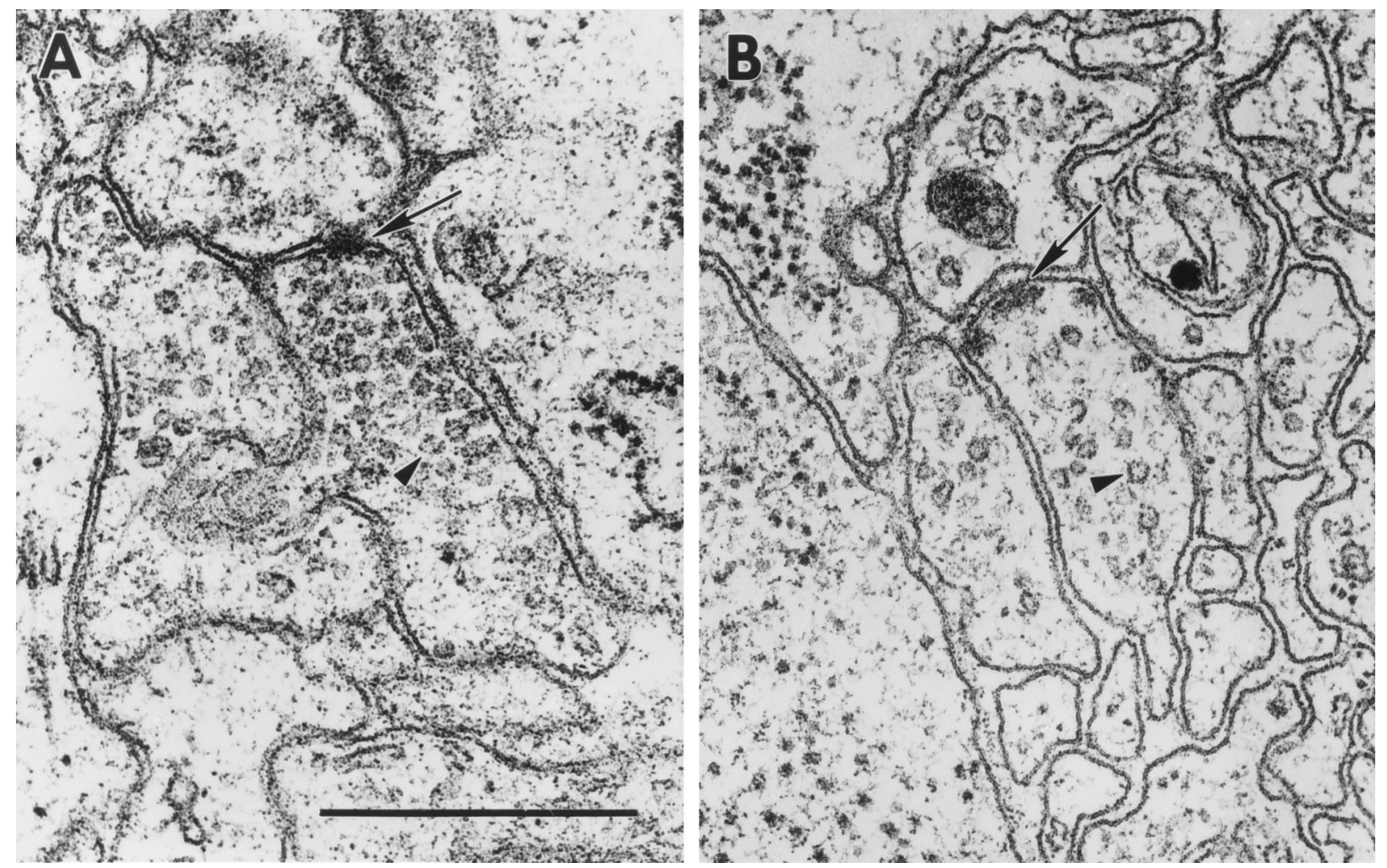

Figure 8. Synaptic vesicle populations are depleted at neuromuscular junctions in $r a b-3$ mutants. Electron micrographs of wild-type $(A)$ and $r a b-3(y 251)$ $(B)$ neuromuscular junctions in the ventral cord. Arrows demarcate the dark thickening of an active zone, and arrowheads identify a typical synaptic vesicle in each photograph. Scale bar, $500 \mathrm{~nm}$.

Table 3. Vesicle populations in $r a b-3$ mutants

\begin{tabular}{lll} 
Genotype & $\begin{array}{l}\text { Synaptic vesicles } \\
\text { per profile }\end{array}$ & NMJ examined \\
\hline N2 (wt) & 38.1 & 15 \\
$r a b-3(y 250)$ & 18.7 & 16 \\
$r a b-3(y 251)$ & 14.4 & 16 \\
$r a b-3(j 449)$ & 17.6 & 20 \\
\hline
\end{tabular}

was relatively consistent among the three $r a b-3$ mutant strains. In the cross section of the axon, the remaining vesicles were not tightly clustered around the presynaptic specialization as they were in wild-type animals (Fig. 8). Furthermore, studies of serial sections of wild-type and js 49 animals revealed that the depletion of synaptic vesicles at the presynaptic specialization is compensated by an increase in vesicles lateral to the active zones; the increase is greater than fivefold at distances $>400 \mathrm{~nm}$ from the presynaptic density (Fig. 9). Despite the diffuse vesicle distribution, total vesicle populations were similar in js49 (9.7 \pm 0.6 vesicles/profile) and the wild type $(8.3 \pm 0.6$ vesicles/profile $)$. Thus, vesicle populations are less tightly clustered around the presynaptic density in $r a b-3$ mutants than in the wild type.

\section{DISCUSSION}

We have isolated nematode mutants that completely lack functional RAB-3. These mutants show few overt behavioral defects and are barely distinguishable from wild-type animals, thereby demonstrating that synaptic transmission is fundamentally intact despite the absence of RAB-3. Our extensive search for $r a b$ molecules failed to identify other $r a b$-3 members that could compensate for the lack of RAB-3. Although proof that $C$. elegans does not contain other rab3 genes will require the complete sequence of the nematode genome, it is extremely unlikely that other rab3 genes are encoded in the genome. Hence, we conclude that RAB-3 is not an essential component of the synaptic transmission apparatus in $C$. elegans.

Despite the relatively normal behavior of $r a b-3$ mutant animals, both synaptic transmission and synaptic morphology are abnormal in the mutants. The transmission defects are most apparent in extracellular recordings from pharyngeal muscles. These recordings document defects in the efficacy of both inhibitory and excitatory synaptic transmission in the pharynx. However, synaptic function seems to be affected more widely, because $r a b-3$ mutants are resistant to the acetylcholinesterase inhibitor aldicarb. Because aldicarb potentiates the action of secreted $\mathrm{ACh}$, the simplest interpretation of this resistance is a general reduction in secretion of ACh from cholinergic neuromuscular junctions. Consistent with such a hypothesis, the morphology of neuromuscular junctions in rab-3 animals is altered. Thus, C. elegans neurons lacking RAB-3 are capable of regulated release, but the efficiency of transmission presumably is reduced because of a decrease in the steady-state population of releasable vesicles at synapses.

The primary morphological defect we observe in $r a b-3$ mutants is that synaptic vesicles are clustered more loosely around synaptic densities than in the wild type. However, vesicle populations in rab-3 animals are not randomly distributed in neurons because synaptic vesicle proteins are absent from commissures and dendrites (assayed immunohistochemically). Furthermore, neither vesicle proteins (assayed immunohistochemically; Fig. 5) nor ves- 


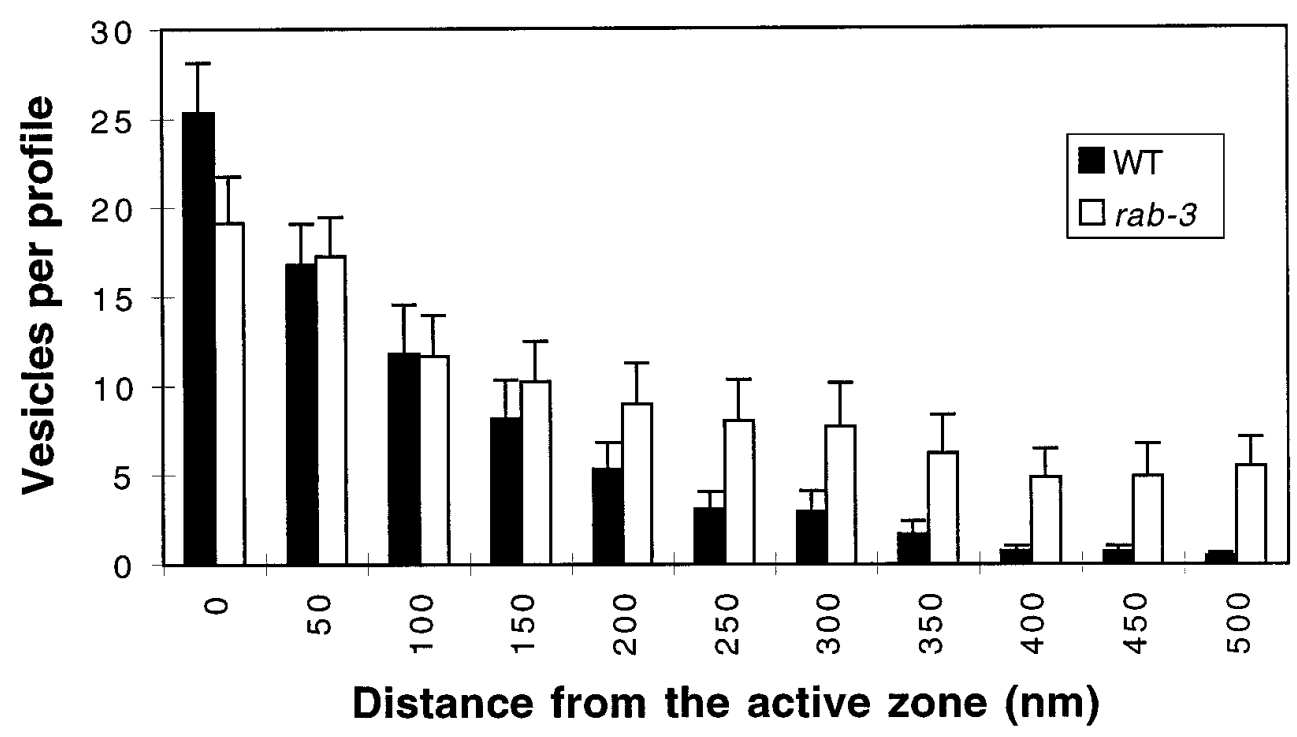

Figure 9. Distribution of vesicles at neuromuscular junctions. Serial section electron micrographs of the ventral cord of wild-type and $r a b-3(j s 49)$ animals were examined. Synaptic vesicles were counted in each motor neuron axonal profile of wild type (black bars; $n=475$ ) and rab-3(js49) (white bars; $n=369$ ). The average number of vesicles is plotted against the distance from the electron-dense specialization found at C. elegans synaptic contacts. Distance was determined by the number of thin sections to the closest active zone in which section thickness was $\sim 50 \mathrm{~nm}$. Error bars are \pm SEM. icles (from ultrastructural data; not shown) accumulate in neuronal cell bodies in rab-3 mutants. These data suggest that trafficking of synaptic vesicles from the soma to synaptic sites is not defective in the absence of RAB-3. Additionally, the size of the total population of synaptic vesicles and the density of synaptic terminals are unchanged. Together these data strongly argue that synaptic development is not perturbed in $r a b-3$ mutants. Thus, the observed morphological defects in vesicle localization are consistent with a synaptic site of action for RAB-3.

The synaptic abnormalities observed in $r a b-3$ mutants could be manifested by a number of different primary defects near the terminal. First, endocytosis of synaptic vesicles might be altered. The rate of endocytosis is unlikely to be perturbed significantly, because vesicle populations are normal. However, in principle, alterations in the site of synaptic vesicle endocytosis could account for the morphological defect we observe. Second, synaptic vesicles could be tethered inefficiently at the synaptic release site in the absence of RAB-3. A pool of tightly clustered vesicles is present at synaptic release sites in most organisms, including $C$. elegans (White et al., 1986). Presumably, this pool is sequestered near the release site by a combination of vesicle-vesicle and vesicle-cytoskeletal interactions. In mammalian cells, expression of mutant forms of rab8 results in reorganization of the cytoskeleton (Peranen et al., 1996). Perhaps RAB-3 regulates interactions between synaptic vesicles and cytoskeletal components in the reserve pool or cytoskeletal "filaments" linking the reserve pool and active zone. Third, RAB-3 could regulate the docking of vesicles at the plasma membrane. This step likely involves the formation of novel contacts with docking proteins. A decrease in the efficiency of docking could account for the ultrastructural defects. In this case, vesicles are mobilized to fill "empty" sites at the active zone but fail to dock efficiently. These "free" vesicle then can drift from the release site to generate the diff use clusters as observed by electron microscopy. However, $r a b-3$ cannot be essential for docking, because release occurs in the absence of the protein. Furthermore, our data are not consistent with a central role for RAB-3 in a postdocking step in vesicle fusion. Synaptic vesicle accumulation at the plasma membrane would be predicted from this type of defect. Indeed, vesicle accumulation is observed in synaptic terminals of C. elegans unc-18 mutants (E. Jorgensen, E. Hartwieg, and H. R. Horvitz, unpublished data). UNC-18 is likely to be a component of the docking and/or fusion machinery (Gengyo-Ando et al., 1993; Pevsner, 1996), because vertebrate homologs interact directly with syntaxin, a component of the proposed synaptic machinery (Hata et al., 1993; Garcia et al., 1994; Pevsner et al., 1994).

The physiological activity of $r a b-3$ mutant synapses is also consistent with there being a defect in vesicle tethering or docking, because transmission is reduced and release appears less synchronous than in wild-type animals. Additionally, mislocalization of ACTH observed in AtT-20 cells expressing rab3 mutants also points to a requirement for $r a b 3$ in regulating the transport or sequestration of vesicles at active zones (Ngsee et al., 1993). A similar dysfunction originally was proposed by Geppert et al. (1994) to explain the electrophysiological defects observed in hippocampal slices isolated from $r a b 3 A$ mutant mice. In these slices repetitive stimulation results in a marked reduction in evoked potentials, suggesting that reduction of the vesicle pool is limiting the exocytic potential of neurons (Geppert et al., 1994). However, additional physiological studies of cultured mouse rab3A mutant hippocampal neurons have demonstrated that vesicle pools are not changed in the mutant and that the depletion on repetitive stimulation results from a larger number of quanta being released during stimulation in the mutant neurons (Geppert et al., 1997). Thus, these data argue that rab3A acts as an inhibitor of release. Our analysis of neuromuscular junctions is not consistent with the observations made at these central synapses, because all of our data point to a reduction in release in rab-3 mutants. It remains a possibility that this difference simply stems from the fact that we examined a neuromuscular synapse rather than a central synapse.

The precise molecular mechanism used by rab proteins in regulating release remains relatively obscure despite a decade of intense study. Rab proteins probably modulate secretion via physical interactions with effector molecules. The regulatory targets of many rab GTPases remain unidentified. The identification and biochemical characterization of these molecules should provide one avenue to delineate the molecular mechanisms underlying the rab regulation of secretion. Additional genetic studies in $C$. elegans such as those used to identify the RAB-3 effector AEX-3 (Iwasaki et al., 1997) provide one of many approaches to solving this complex cell biology problem. 


\section{REFERENCES}

Avery L (1993) Motor neuron M3 controls pharyngeal muscle relaxation timing in Caenorhabditis elegans. J Exp Biol 175:283-297.

Avery L, Raizen D, Lockery S (1995) Electrophysiological methods. In: Methods in cell biology, Vol 48, Caenorhabditis elegans: modern biological analysis of an organism (Epstein HF, Shakes DC, eds), pp 251-269. San Diego: Academic.

Bargmann CI, Hartwieg E, Horvitz HR (1993) Odorant-selective genes and neurons mediate olfaction in C. elegans. Cell 74:515-527.

Bennett MK, Scheller RH (1994) A molecular description of synaptic vesicle membrane trafficking. Annu Rev Biochem 63:63-100.

Blumenthal $\mathrm{T}$ (1995) Trans-splicing and polycistronic transcription in Caenorhabditis elegans. Trends Genet 11:132-136.

Bourne HR, Sanders DA, McCormick F (1991) The GTPase superfamily: conserved structure and molecular mechanism. Nature 349:117-127.

Coulson A, Sulson J, Brenner S, Karn J (1986) Toward a physical map of the Caenorhabditis elegans genome. Proc Natl Acad Sci USA 83:7821-7825.

Coulson A, Waterston R, Kiff J, Sulston J, Kohara Y (1988) Genome linking with yeast artificial chromosomes. Nature 335:184-186.

Devereux J, Haeberli P, Smithies O (1984) A comprehensive set of sequence analysis programs for the VAX. Nucleic Acids Res 12:387-395.

Ferro-Novick S, Novick P (1993) The role of GTP-binding proteins in transport along the exocytic pathway. Annu Rev Cell Biol 9:575-599.

Fire A, Harrison SW, Dixon D (1990) A modular set of lacZ fusion vectors for studying gene expression in Caenorhabditis elegans. Gene 93:189-198.

Fischer von Mollard G, Mignery GA, Baumert M, Perin MS, Hanson TJ, Burger PM, Jahn R, Sudhof TC (1990) Rab3 is a small GTP-binding protein exclusively localized to synaptic vesicles. Proc Natl Acad Sci USA 87:1988-1992.

Fischer von Mollard G, Stahl B, Khokhlatchev A, Sudhof TC, Jahn R (1994a) Rab3C is a synaptic vesicle protein that dissociates from synaptic vesicles after stimulation of exocytosis. J Biol Chem 269:10971-10974.

Fischer von Mollard G, Stahl B, Li C, Sudhof TC, Jahn R (1994b) Rab proteins in regulated exocytosis. Trends Biochem Sci 19:164-168.

Fitch DHA, Bugaj-Gaweda B, Emmons SW (1995) 18S ribosomal RNA gene phylogeny for some Rhabditiae related to Caenorhabditis. Mol Biol Evol 12:346-358.

Garcia EP, Gatti E, Butler M, Burton J, De Camilli P (1994) A rat brain Sec1 homologue related to Rop and UNC18 interacts with syntaxin Proc Natl Acad Sci USA 91:2003-2007.

Gengyo-Ando K, Kamiya Y, Yamakawa A, Kodaira K, Nishiwaki K, Miwa J, Hori I, Hosono R (1993) The C. elegans unc-18 gene encodes a protein expressed in motor neurons. Neuron 11:703-711.

Geppert M, Bolshakov VY, Siegelbaum SA, Takei K, De Camilli P, Hammer RE, Sudhof TC (1994) The role of Rab3A in neurotransmitter release. Nature 369:493-497.

Geppert M, Goda Y, Stevens C, Sudhof TC (1997) The small GTPbinding protein rab3A regulates a late step in synaptic vesicle fusion. Nature 387:810-814.

Hall DH, Hedgecock EM (1991) Kinesin-related gene unc-104 is required for axonal transport of synaptic vesicles in $C$. elegans. Cell 65:837-847.

Hata Y, Slaughter CA, Sudhof TC (1993) Synaptic vesicle fusion complex contains unc-18 homologue bound to syntaxin. Nature 366:347-351.

Herman RK, Horvitz HR (1980) Genetic analysis of Caenorhabditis elegans. In: Nematodes as biological models, Vol 1, Behavioral and developmental models (Zuckerman BM, ed), pp 227-262. New York: Academic.

Hodgkin J (1983) Male phenotypes and mating efficiency in C. elegans. Genetics 103:43-64.

Holz R, Brondyk W, Senter R, Kuizon L, Macara I (1994) Evidence for the involvement of Rab3A in $\mathrm{Ca}^{2+}$-dependent exocytosis from adrenal chromaffin cells. J Biol Chem 269:10229-10234.

Horvitz HR, Brenner S, Hodgkin J, Herman RK (1979) A uniform genetic nomenclature for the nematode Caenorhabditis elegans. Mol Gen Genet 175:129-133.

Hosono R, Kamiya Y (1991) Additional genes result in an elevation of acetylcholine levels by mutations in Caenorhabditis elegans. Neurosci Lett 128:243-244.

Hosono R, Sassa T, Kuno S (1987) Mutations affecting acetylcholine levels in the nematode Caenorhabditis elegans. J Neurochem 49:1820-1823.

Innis MA, Gelfand DH, Sninsky JJ, White TJ, editors (1990) PCR protocols: a guide to methods and applications. San Diego: Academic.

Iwasaki K, Staunton J, Nonet ML, Thomas J (1997) aex-3 encodes a novel gene regulator of presynaptic activity in $C$. elegans. Neuron 18:613-622.

Johannes L, LLedo PM, Roa M, Vincent JD, Henry JP, Darchem F (1994) The GTPase rab3a negatively controls calcium-dependent exocytosis in neuroendocrine cells. EMBO J 13:2029-2037.

Johnston PA, Archer BT, Robinson K, Mignery GA, Jahn R, Sudhof TC (1991) Rab3A attachment to the synaptic membrane mediated by a conserved polyisoprenylated carboxyl-terminal sequence. Neuron 7:101-109.

Lewis JA, Wu CH, Levine JH, Berg H (1980) Levamisole-resistant mutants of the nematode Caenorhabditis elegans appear to lack pharmacological acetylcholine receptors. Neuroscience 5:967-989.

Lian J, Stone S, Jiang Y, Lyons P, Ferro-Novick S (1994) Ypt1p implicated in v-SNARE activation. Nature 372:698-701.

Liu DW, Thomas JH (1994) Regulation of a periodic motor program in C. elegans. J Neurosci 14:1953-1962.

Lledo P, Vernier P, Vincent J, Mason W, Zorec R (1993) Inhibition of Rab3B expression attenuates $\mathrm{Ca}^{2+}$-dependent exocytosis in anterior pituitary cells. Nature 364:540-544.

McCaman RE, Stetzler J (1977) Radiochemical assay for ACh: modifications for sub-picomole measurements. J Neurochem 28:669-671.

McIntire SL, Garriga G, White J, Jacobson D, Horvitz HR (1992) Genes necessary for directed axonal elongation or fasciculation in C. elegans. Neuron 8:307-322.

Mello CC, Kramer JM, Stinchcomb D, Ambros V (1991) Efficient gene transfer in C. elegans: extrachromosomal maintenance and integration of transforming sequences. EMBO J 10:3959-3970.

Miller L (1991) Sex determination and dosage compensation in C. elegans. PhD thesis, Massachusetts Institute of Technology.

Mizoguchi A, Kim S, Ueda T, Kikuchi A, Yorifuji H, Hirokawa N, Takai Y (1990) Localization and subcellular distribution of smg p25A, a ras p21-like GTP-binding protein, in rat brain. $\mathrm{J}$ Biol Chem 265:11872-11879.

Ngsee JK, Fleming AM, Scheller RH (1993) A rab protein regulates the localization of secretory granules in AtT-20 cells. Mol Biol Cell 4:747-756.

Nguyen M, Alfonso A, Johnson CD, Rand JB (1995) Caenorhabditis elegans mutants resistant to inhibitors of acetylcholinesterase. Genetics 140:527-535.

Nonet ML, Meyer BJ (1991) Early aspects of Caenorhabditis elegans sex determination and dosage compensation are regulated by a zinc-finger protein. Nature 351:65-68.

Nonet ML, Grundahl K, Meyer BJ, Rand JB (1993) Synaptic function is impaired but not eliminated in C. elegans mutants lacking synaptotagmin. Cell 73:1291-1305.

Nuoffer C, Blach WE (1994) GTPases: multifunctional molecular switches regulating vesicular traffic. Annu Rev Biochem 63:949-990.

Oberhause A, Monck J, Balch W, Fernandez J (1992) Exocytotic fusion is activated by Rab3a peptides. Nature 360:270-273.

Okada Y, Yamazaki H, Sekine-Aizawa Y, Hirokawa N (1995) The neuron-specific kinesin superfamily protein KIF1A is a unique monomeric motor for anterograde axonal transport of synaptic vesicle precursors. Cell 81:769-780.

Padfield P, Balch W, Jamieson J (1992) A synthetic peptide of the rab3a effector domain stimulates amylase release from permeabilized pancreatic acini. Proc Natl Acad Sci USA 89:1656-1660.

Peranen J, Auvinen P, Virta H, Wepf R, Simons K (1996) Rab8 promotes polarized membrane transport through reorganization of actin and microtubules in fibroblasts. J Cell Biol 135:153-167.

Pevsner J (1996) The role of Sec1p-related proteins in vesicle trafficking in the nerve terminal. J Neurosci Res 45:89-95.

Pevsner J, Hsu SC, Scheller RH (1994) n-Sec1: a neural-specific syntaxin-binding protein. Proc Natl Acad Sci USA 91:1445-1449.

Piiper A, Stryjek-Kaminska D, Zeuzem S (1994) Synthetic Rab3A effector domain peptide stimulates inositol 1,4,5-trisphosphate production in various permeabilized cells. Biochem Biophys Res Commun 203:756-762. 
Raizen DM, Avery L (1994) Electrical activity and behavior in the pharynx of Caenorhabditis elegans. Neuron 12:483-495.

Raizen DM, Lee RYN, Avery L (1995) Interacting genes required for pharyngeal excitation by motor neuron MC in Caenorhabditis elegans. Genetics 141:1365-1382.

Sambrook J, Fritsch EF, Maniatis T (1989) Molecular cloning: a laboratory manual, 2nd Ed. Cold Spring Harbor, NY: Cold Spring Harbor Laboratory.

Simons K, Zerial M (1993) Rab proteins and the road maps for intracellular transport. Neuron 11:789-799.

Sogaard M, Tani K, Ye RR, Geromanos S, Tempst P, Kirchhausen T, Rothman JE, Sollner T (1994) A rab protein is required for the assembly of SNARE complexes in the docking of transport vesicles. Cell 78:937-948.

Sollner T, Rothman JE (1994) Neurotransmission: harnessing fusion machinery at the synapse. Trends Neurosci 17:344-348.

Sudhof T (1995) The synaptic vesicle cycle: a cascade of protein-protein interactions. Nature 375:645-653.

Sulston J, Hodgkin J (1988) Methods. In: The nematode Caenorhabditis elegans (Wood WB, ed), pp 587-606. Cold Spring Harbor, NY: Cold Spring Harbor Laboratory.

White JG, Southgate E, Thomson JN, Brenner S (1986) The structure of the nervous system of Caenorhabditis elegans. Philos Trans R Soc Lond [Biol] 314:1-340. 